\title{
Specificity in communities of Symbiodinium in corals from Johnston Atoll
}

\author{
Michael Stat ${ }^{*}$, Xavier Pochon, Rebecca O. M. Cowie, Ruth D. Gates \\ Hawaii Institute of Marine Biology, School of Ocean and Earth Science and Technology, University of Hawaii, \\ 46-007 Lilipuna Rd, Kaneohe, Hawaii 96744, USA
}

\begin{abstract}
The diversity of endosymbiotic dinoflagellates (Symbiodinium) in corals at Johnston Atoll in the central Pacific Ocean was assessed using both the internal transcribed spacer 2 (ITS2) region of the nuclear rDNA and chloroplast 23S rDNA. More sequences were recovered from corals using the ITS2 primers than with the chloroplast 23S primers, a finding that reflects both the higher taxonomic resolution and level of intragenomic variation in ITS2 in eukaryotes as compared to chloroplast 23S. Parsimony network analysis, Bray-Curtis coefficient of similarity and 1-way analysis of similarity resolved coral species- and/or genus-specific lineages and/or groupings of Symbiodinium that were generally congruent between the 2 genetic markers. Comparison of coral-Symbiodinium assemblages at Johnston Atoll with those in corals sampled on other reefs in the Pacific reveals differences that include novel host-symbiont unions and a Symbiodinium lineage previously reported to be Caribbean-specific in Acropora from Johnston Atoll.
\end{abstract}

KEY WORDS: Symbiodinium $\cdot$ Coral $\cdot$ Dinoflagellate $\cdot$ Symbiosis $\cdot$ ITS $2 \cdot$ Chloroplast

\section{INTRODUCTION}

Dinoflagellates in the genus Symbiodinium are found in symbiosis with a diverse range of marine invertebrate taxa and some protists (Lobban et al. 2002, reviewed in Coffroth \& Santos 2005, Stat et al. 2006). In corals, Symbiodinium reside inside host gastroderm cells and supply the animal with organic carbon fixed via photosynthesis, a resource that underpins the growth and formation of coral reefs (Muscatine et al. 1981). The Symbiodinium genus is currently differentiated into 8 phylogenetic groups, clades $\mathrm{A}$ to $\mathrm{H}$, based on nuclear 18S and 28S rDNA (Rowan \& Powers 1991, Carlos 1999, LaJeunesse \& Trench 2000, LaJeunesse 2001, Pawlowski et al. 2001, Pochon et al. 2001, 2004) and the chloroplast 23S domain V (Santos et al. 2002, Pochon et al. 2006). Each of these clades is further divided into subclade genetic types based on more variable markers such as the internal transcribed spacer regions of the nuclear rDNA (ITS1 and ITS2, e.g. van Oppen et al. 2001, 2005, LaJeunesse 2002, 2005,
LaJeunesse et al. 2003, 2004a, Pochon et al. 2007). Patterns in Symbiodinium subclade types associate with biogeography, host species, light environment and Symbiodinium acquisition strategy (e.g. Rowan \& Knowlton 1995, Baker et al. 1997, Rowan et al. 1997, Rodriguez-Lanetty et al. 2001, Ulstrup \& van Oppen 2003, Iglesias-Prieto et al. 2004, LaJeunesse 2005, Stat et al. 2008a). The taxonomy of Symbiodinium and the specificity of the association between hosts and Symbiodinium clades and subclade types can often explain differences in coral physiology such as growth rate, thermal tolerance, photophysiology and bleaching and disease susceptibility (Rowan \& Knowlton 1995, Rowan et al. 1997, Baker et al. 2004, Little et al. 2004, Rowan 2004, Tchernov et al. 2004, Sampayo et al. 2008, Stat et al. 2008b, Correa et al. 2009).

The eukaryotic nuclear rDNA region is repeated multiple times within a genome (e.g. the dinoflagellate Prorocentrum micans contains 200 copies; Long \& Dawid 1980, Herzog \& Maroteaux 1986). The ITS regions of the rDNA are more variable than the $18 \mathrm{~S}$, 
28S and 5.8S regions and are often used for distinguishing more closely related taxa (Coleman 2003); however, the rapid rate of evolution in the ITS regions combined with high levels of repetition in rDNA drive intragenomic and intraspecific variation (Adachi et al. 1996, Le Blancq et al. 1997, Lott et al. 1998, Coleman 2003, Denboh et al. 2003, Litaker et al. 2003, 2007, Orsini et al. 2004, Rynearson \& Armbrust 2004, Shankle et al. 2004, Godhe et al. 2006, Aktas et al. 2007, Chen et al. 2007, Kawahata et al. 2007, Cantacessi et al. 2008). Intragenomic variation has been demonstrated in cultured Symbiodinium cells and is likely a confounding factor in accurately assessing diversity in symbiotic communities (Thornhill et al. 2007). An endosymbiotic lifestyle may also influence mutation rates for Symbiodinium as compared to free-living dinoflagellates, resulting in less divergence between species (Litaker et al. 2007). The evolutionary time required for concerted evolution to act on the genome and patterns in the ecological distribution of some Symbiodinium ITS2 types have been used as evidence to suggest that the ITS2 provides species-level taxonomic resolution (e.g. LaJeunesse 2001, Sampayo et al. 2009). However, whether single base changes within the dominant sequence across the ITS2 region of the rDNA consistently represent different Symbiodinium species remains contentious and clusters of closely related sequences have also been interpreted as ecotypes (Correa \& Baker 2009). In other species of diatoms and dinoflagellates, patterns in the biogeography and ecology of different ITS2 sequences have been interpreted as variation at the subspecies level (Rynearson \& Armbrust 2004, Shankle et al. 2004, Godhe et al. 2006). This genetic diversity and accompanying physiological diversity within a species is explained as plasticity that allows the species to occupy a wide geographic and environmental gradient.

Genes in the chloroplast genome of dinoflagellates exist on individual plastid minicircles (Gray 1999, Zhang et al. 1999, Green 2004). The copy number of these minicircles is unknown; however, some dinoflagellates show evidence of multiple copies (Zhang et al. 2002, Koumandou \& Howe 2007). The chloroplast 23S rDNA is considered to have a relatively fast rate of DNA evolution and is therefore a potentially useful marker for investigating Symbiodinium diversity; however, to date it has not been applied broadly to the genus (but see Santos et al. 2002, Pochon et al. 2006).

In the present study, the ITS2 and chloroplast 23S rDNA markers were used to explore Symbiodinium diversity in corals from Johnston Atoll. Johnston Atoll, situated southwest of the main Hawaiian Islands in the central Pacific Ocean, represents one of the most isolated reef ecosystems in the world and an area where Symbiodinium diversity has not been examined before. The aims of the present study were to: (1) assess the diversity of Symbiodinium in 12 coral species using ITS2 and chloroplast 23S, (2) examine host specificity in coralSymbiodinium associations from this area, (3) compare the Symbiodinium diversity recovered from corals at Johnston Atoll with the diversity found in corals elsewhere in the Pacific, and (4) compare the utility of the ITS2 and chloroplast 23S markers in assessing Symbiodinium diversity and patterns of specificity.

\section{MATERIALS AND METHODS}

Sample collection. Five colonies of each of 12 coral species $(\mathrm{n}=60$ colonies) were sampled from 9 sites around the remote coral reef ecosystem at Johnston Atoll in June 2006 (Fig. 1, Table 1). Coral fragments $(\approx 2 \mathrm{~mm}$ of tissue) were stored in $400 \mu \mathrm{l}$ of DNA extraction buffer $(50 \% \mathrm{w} / \mathrm{v}$ guanidinium isothiocyanate; $50 \mathrm{mM}$ Tris pH 7.6; $10 \mu \mathrm{M}$ EDTA; $4.2 \% \mathrm{w} / \mathrm{v}$ sarkosyl; $2.1 \% \mathrm{v} / \mathrm{v} \beta$-mercaptoethanol) until further processing.

DNA extraction, PCR, cloning and sequencing. For extraction of nucleic acids, samples were incubated at $72^{\circ} \mathrm{C}$ for $10 \mathrm{~min}$, centrifuged at $16000 \times g$ for $5 \mathrm{~min}$ and the resulting supernatant mixed with an equal volume of isopropanol and incubated at $-20^{\circ} \mathrm{C}$ overnight. The DNA was precipitated by centrifugation at $16000 \times g$ for $15 \mathrm{~min}$ and the DNA pellet washed in $70 \%$ ethanol and resuspended and stored in Tris buffer $(0.1 \mathrm{M} \mathrm{pH} 9)$.

Partial 5.8S, the entire ITS2 and partial 28S rDNA genes in Symbiodinium were amplified in PCR using itsD (Pochon et al. 2001; forward; 5'-GTG AAT TGC AGA ACT CCG TG-3', 5 pmol) and its2rev2 (reverse; 5'-CCT CCGCTTACT TATATGCTT-3', 5 pmol) primers and $0.5 \mathrm{U}$ of Immolase (Bioline) in a $25 \mu \mathrm{l}$ reaction using the following cycling conditions: denaturation at $95^{\circ} \mathrm{C}$ for 7 min followed by 35 cycles of $45 \mathrm{~s}$ at $95^{\circ} \mathrm{C}$, $45 \mathrm{~s}$ at $52^{\circ} \mathrm{C}, 45 \mathrm{~s}$ at $72^{\circ} \mathrm{C}$ and a final extension at $72^{\circ} \mathrm{C}$ for $5 \mathrm{~min}$. The 23S-rDNA domain V region of the Symbiodinium chloroplast was amplified in PCR using the $23 \mathrm{~S} 1$ (forward; 5'-GGC TGT AAC TAT AAC GGT CC-3',

Table 1. Collection sites at Johnston Atoll where corals were sampled for Symbiodinium genotyping

\begin{tabular}{|lccc|}
\hline Site & Depth $(\mathrm{m})$ & Latitude & Longitude \\
\hline JA-HIMB3 & $3-7$ & $16^{\circ} 44.576 \mathrm{~N}$ & $169^{\circ} 32.269 \mathrm{~W}$ \\
JOH-1AP & $15-20$ & $16^{\circ} 47.162 \mathrm{~N}$ & $169^{\circ} 27.710 \mathrm{~W}$ \\
JOH-20 & $5-10$ & $16^{\circ} 45.670 \mathrm{~N}$ & $169^{\circ} 31.618 \mathrm{~W}$ \\
JOH-22 & $10-15$ & $16^{\circ} 45.858 \mathrm{~N}$ & $169^{\circ} 31.535 \mathrm{~W}$ \\
JOH-23 & $2-4$ & $16^{\circ} 44.838 \mathrm{~N}$ & $169^{\circ} 30.559 \mathrm{~W}$ \\
JOH-25 & $7-12$ & $16^{\circ} 46.615 \mathrm{~N}$ & $169^{\circ} 29.502 \mathrm{~W}$ \\
JOH-4P & $3-7$ & $16^{\circ} 46.260 \mathrm{~N}$ & $169^{\circ} 30.336 \mathrm{~W}$ \\
JOH-5P & $2-4$ & $16^{\circ} 46.449 \mathrm{~N}$ & $169^{\circ} 29.821 \mathrm{~W}$ \\
JOH-6P & $3-7$ & $16^{\circ} 43.111 \mathrm{~N}$ & $169^{\circ} 33.080 \mathrm{~W}$ \\
\hline
\end{tabular}




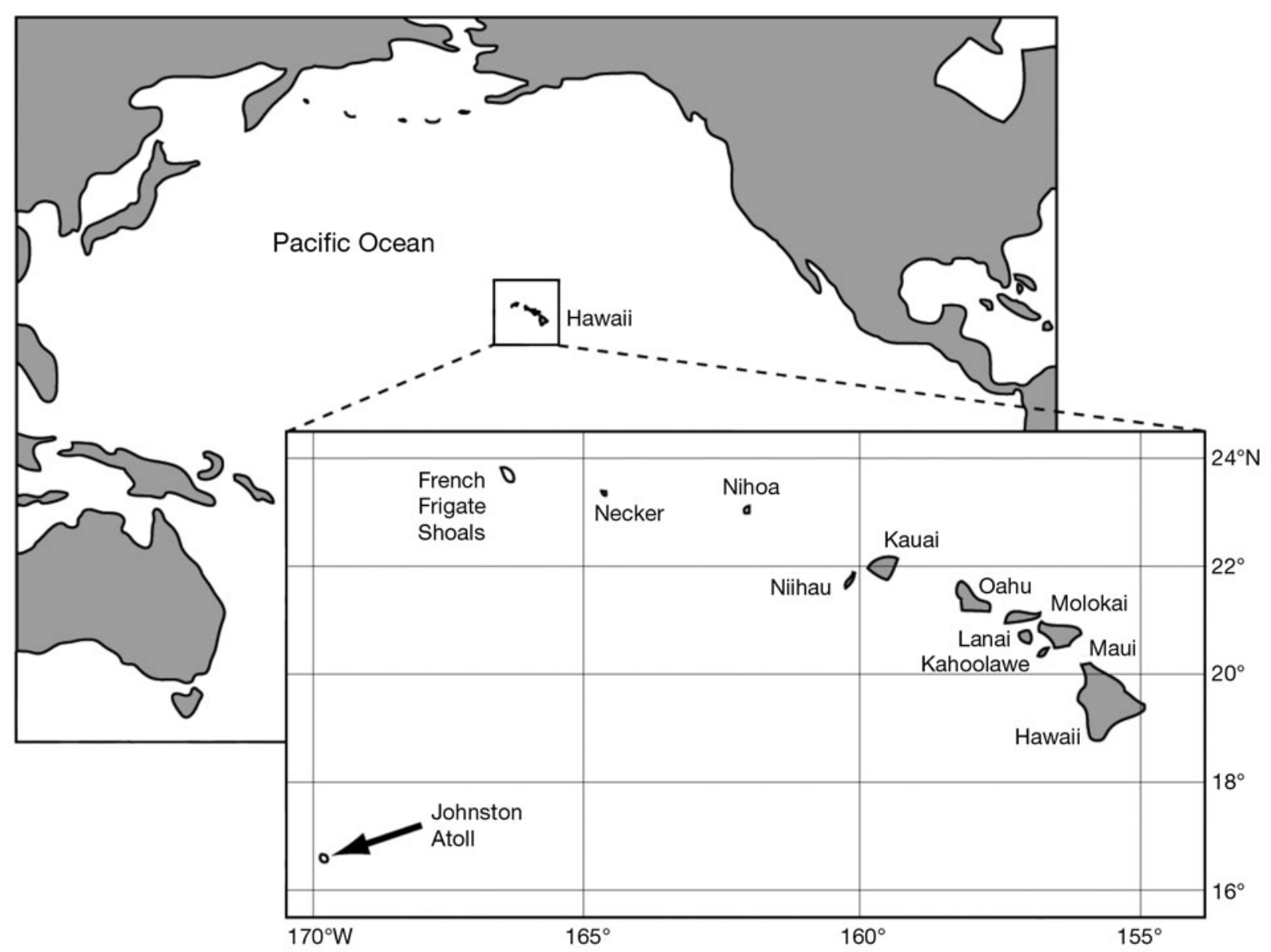

Fig. 1. Location of Johnston Atoll (indicated by arrow) where corals were sampled for Symbiodinium genotyping

5 pmols) and 23S2 (reverse; 5'-CCA TCG TAT TGA ACC CAGC-3' 5 pmols) primers (Zhang et al. 2000) and $0.5 \mathrm{U}$ of Immolase (Bioline) in a $25 \mu \mathrm{l}$ reaction as described above with an annealing temperature of $55^{\circ} \mathrm{C}$. PCR amplicons were purified using the QIAquick $^{\circledR}$ PCR Purification Kit (Qiagen), ligated into the pGEM $^{\circledR}-\mathrm{T}$ Easy vector (Promega), transformed into $\alpha$-select gold efficiency competent cells (Bioline) and grown overnight in Circlegrow ${ }^{\circledR}$ (MP Biomedicals). Plasmids were purified using the Perfectprep ${ }^{\circledR}$ Plasmid Isolation Kit (Eppendorf) and at least 10 clones from each PCR product cycle-sequenced using BigDye Terminators (PerkinElmer) on an ABI-3100 automated sequencer at the University of Hawaii. Ambiguous sequences were confirmed by alignment to the reverse complement.

Sequence and phylogenetic analyses. Sequences were inspected, aligned and edited using the software MacVector $^{\circledR}$ 10. Identical sequences obtained from more than one clone library and singletons matching existing GenBank entries were used in downstream analysis. The remaining clone singletons were assu- med to be artifacts or rare intragenomic repeats not useful for diversity analysis, and the nucleotide at the site of polymorphism converted to the consensus. In addition, IT2S folding was conducted using previously published Symbiodinium ITS2 structures as templates (Hunter et al. 2007, Thornhill et al. 2007) in the ITS2 database interactive website (Schultz et al. 2006, Selig et al. 2008) and manually edited using the software 4SALE (Seibel et al. 2006, 2008). Potential pseudogenes were characterized by significant changes to the 5.8S sequence not observed in Symbiodinium or other closely related dinoflagellates (Thornhill et al. 2007) and changes to the secondary structure of the ITS2 RNA molecule that likely disrupt the functional fold. As for singletons above, sequences representing putative pseudogenes were converted to the consensus at the site of polymorphism. Editing singletons and potential pseudogenes in this way collapsed minor sequence variants to the closest dominant sequence present in the data set determined from statistical parsimony. Published Symbiodinium ITS2 sequences were named as in previous studies (LaJeunesse 2001, 
2002, 2005, LaJeunesse et al. 2003, 2004b). Novel ITS2 sequences were assigned a unique specifier reflecting the closest relationship to a published dominant subclade type. Novel sequences were assigned a decimal and a numeral to distinguish them from the published type and each other. For example, 3 different sequences closely related to $\mathrm{C} 15$ were named C15.1, C15.2, and C15.3. For chloroplast 23S, sequences were named numerically with a letter corresponding to the Symbiodinium clade followed by a $p$ to reflect the plastid origin of the sequence, and numbered sequentially.

Statistical parsimony networks were run using the software TCS v.1.21 (Clement et al. 2000). The cladogram estimation was performed under a $95 \%$ connection limit and gaps were treated as a 5th state with the alignment edited so that the entire indel was considered a single mutation.

Symbiodinium sequence signatures and community analyses. All edited Symbiodinium sequences recovered from an individual coral colony are referred to here as the sequence signature of that colony. Symbiodinium sequence signatures from all coral colonies were compared using the Bray-Curtis coefficient of similarity $(S)$ in the software package PRIMER v.6 (Clarke \& Warwick 2001), which ranges between 0 and 100, where a value of 100 indicates an identical assemblage (Bray \& Curtis 1957). For both the ITS2 and chloroplast 23S rDNA, the Symbiodinium sequence signature in each coral was first standardized by square-root transforming the relative abundance of each sequence in that colony prior to calculation of $S$. To test for the relationship of Symbio- dinium sequence signatures in the coral colonies from Johnston Atoll, Bray-Curtis similarities were analyzed by hierarchical clustering in PRIMER, and the SIMPROF test conducted to establish the significance of the dendrogram nodes.

Symbiodinium sequence signatures were grouped by coral species to obtain a community sequence signature for each coral species. To test for host specificity, 1-way analysis of similarities (ANOSIM) was calculated in PRIMER to detect significant differences in the Symbiodinium community sequence signature for each marker (ITS2 and chloroplast 23S) grouped by coral species (global test) and between coral species (pairwise test). The test statistic (R) in ANOSIM ranges between +1 to -1 , where a value of 0 indicates no differences between groups, values approaching +1 indicate partitioning of variation by group and values approaching -1 indicate partitioning of variation within each group is greater than between groups.

\section{RESULTS}

\section{Symbiodinium sequence diversity}

A total of 615 Symbiodinium ITS2 sequences and 602 Symbiodinium chloroplast 23S sequences were recovered from the coral colonies sampled at Johnston Atoll and the same Symbiodinium clades were detected using both markers (Table 2). Symbiodinium clade A represented 2.8 and $3.5 \%$, clade C 85.2 and $83.2 \%$, and clade D 12.0 and $13.3 \%$ of the ITS2 and chloroplast 23S clone

Table 2. Symbiodinium ITS2 and chloroplast 23S sequences identified in the corals sampled at Johnston Atoll and from other studies in the Pacific. Numbers superscript and in parentheses indicate the frequency of that sequence recovered from the coral. Numbers subscript refer to source. AS: American Samoa; GBR: Great Barrier Reef; NWHI: Northwestern Hawaiian Islands

\begin{tabular}{|c|c|c|c|c|}
\hline Coral host & Site & $\begin{array}{c}\text { ITS2 } \\
\text { signature }\end{array}$ & $\begin{array}{c}\text { 23S } \\
\text { signature }\end{array}$ & Pacific-wide \\
\hline $\begin{array}{l}\text { Acroporidae } \\
\text { Acropora cytherea }\end{array}$ & $\begin{array}{l}\text { JOH-20 } \\
\text { JOH-23 } \\
\text { JOH-4P } \\
\text { JOH-5P } \\
\text { JOH-6P }\end{array}$ & $\begin{array}{c}\mathrm{C} 1^{(2)}, \mathrm{C} 3^{(4)}, \mathrm{C} 3.2^{(2)}, \mathrm{C} 3.8^{(1)}, \mathrm{C} 3.9^{(1)}, \mathrm{C} 3.11^{(1)}, \mathrm{C} 3 \mathrm{~b}^{(1)} \\
\mathrm{C} 1^{(3)}, \mathrm{C} 3^{(7)}, \mathrm{C} 3.2^{(1)} \\
\mathrm{C} 1^{(2)}, \mathrm{C} 3^{(3)}, \mathrm{C} 3.2^{(2)}, \mathrm{C} 3.7^{(1)}, \mathrm{C} 3.8^{(1)}, \mathrm{C} 3.9^{(1)}, \mathrm{C} 3.10^{(1)} \\
\mathrm{C} 1^{(1)}, \mathrm{C} 3^{(8)}, \mathrm{C} 3.2^{(1)}, \mathrm{C} 3.8^{(1)} \\
\mathrm{C} 1^{(2)}, \mathrm{C} 3^{(5)}, \mathrm{C} 3.10^{(1)}, \mathrm{C} 3.11^{(1)}, \mathrm{C} 21.13^{(2)}\end{array}$ & $\begin{array}{l}\mathrm{C} p 4^{(10)} \\
\mathrm{Cp} 4^{(10)} \\
\mathrm{Cp} 4^{(10)} \\
\mathrm{Cp} 4^{(11)} \\
\mathrm{Cp} 4^{(11)}\end{array}$ & $\begin{array}{c}\text { Acropora: } \\
\text { GBR }_{1,2,3,4} \\
\text { A1, C1, C3, C3h, } \\
\text { C3i, C3k, D1, D2 }\end{array}$ \\
\hline Acropora nasuta & $\begin{array}{l}\text { JOH-1AP } \\
\text { JOH-20 } \\
\text { JOH-4P } \\
\text { JOH-4P } \\
\text { JOH-4P }\end{array}$ & $\begin{array}{c}\mathrm{C} 1^{(1)}, \mathrm{C} 3^{(7)}, \mathrm{C} 3.2^{(1)}, \mathrm{C} 3.8^{(1)}, \mathrm{C} 21.12^{(2)} \\
\mathrm{C} 1^{(5)}, \mathrm{C} 3^{(3)}, \mathrm{C} 3.7^{(1)}, \mathrm{C} 3 \mathrm{~b}^{(1)} \\
\mathrm{C} 1^{(3)}, \mathrm{C} 3^{(4)}, \mathrm{C} 3.2^{(1)}, \mathrm{C} 3 \mathrm{~b}^{(1)}, \mathrm{C} 21.12^{(1)}, \mathrm{C} 21.13^{(1)} \\
\mathrm{C} 1^{(3)}, \mathrm{C} 3^{(3)}, \mathrm{C} 3.8^{(1)}, \mathrm{C} 3 \mathrm{~b}^{(2)}, \mathrm{C} 21.13^{(1)} \\
\mathrm{C} 1^{(1)}, \mathrm{C} 3^{(4)}, \mathrm{C} 3.2^{(2)}{ }^{\prime}, \mathrm{C} 3.8^{(1)}, \mathrm{C} 3.10^{(1)}, \\
\mathrm{C} 3.11^{(1)}, \mathrm{C} 3 \mathrm{~b}^{(1)}, \mathrm{C} 21.12^{(1)}\end{array}$ & $\begin{array}{l}\mathrm{Cp} 4^{(10)} \\
\mathrm{C} p 4^{(10)} \\
\mathrm{C} p 4^{(10)} \\
\mathrm{Cp} 4^{(10)} \\
\mathrm{C} p 4^{(10)}\end{array}$ & $\begin{array}{c}\mathrm{Japan}_{2} \\
\mathrm{C} 1, \mathrm{C} 3, \mathrm{C} 3 \mathrm{i} \\
\mathrm{NWHI}_{5} \\
\mathrm{~A} 1, \mathrm{C} 1 \mathrm{c}, \mathrm{C} 27\end{array}$ \\
\hline Montipora capitata & $\begin{array}{l}\text { JA-HIMB3 } \\
\text { JA-HIMB3 } \\
\text { JOH-20 } \\
\text { JOH-20 } \\
\text { JOH-6P }\end{array}$ & $\begin{array}{c}\mathrm{C} 17^{(2)}, \mathrm{C} 17.2^{(2)}, \mathrm{C} 31^{(5)}, \mathrm{D} 1 \mathrm{a}^{(1)} \\
\mathrm{D} 1^{(5)}, \mathrm{D} 1 \mathrm{a}^{(5)} \\
\mathrm{C} 17.2^{(3)}, \mathrm{C} 21^{(1)}, \mathrm{C} 31^{(4)}, \mathrm{D} 1^{(1)}, \mathrm{D} 1 \mathrm{a}^{(1)} \\
\mathrm{C} 17^{(1)}, \mathrm{C} 17.2^{(4)}, \mathrm{C} 21^{(1)}, \mathrm{C} 31^{(4)} \\
\mathrm{D} 1^{(3)}, \mathrm{D} 1 \mathrm{a}^{(7)}\end{array}$ & $\begin{array}{l}\mathrm{C} p 7^{(5)}, \mathrm{D} p 1^{(5)} \\
\mathrm{D} p 1^{(10)} \\
\mathrm{C} p 7^{(2)}, \mathrm{D} p 1^{(8)} \\
\mathrm{C} p 7^{(10)} \\
\mathrm{D} p 1^{(10)}\end{array}$ & $\begin{array}{c}\text { Montipora: } \\
\text { GBR }_{1,2,4} \\
\text { C15, C17, C21, C31 } \\
\\
\text { Hawaii } \\
\text { C31, D1a }\end{array}$ \\
\hline
\end{tabular}


Table 2 (continued)

\begin{tabular}{|c|c|c|c|c|}
\hline Coral host & Site & $\begin{array}{c}\text { ITS2 } \\
\text { signature }\end{array}$ & $\begin{array}{c}\text { 23S } \\
\text { signature }\end{array}$ & Pacific-wide \\
\hline Montipora patula & $\begin{array}{l}\text { JA-HIMB3 } \\
\text { JA-HIMB3 } \\
\text { JOH-20 } \\
\text { JOH-23 } \\
\text { JOH-4P }\end{array}$ & $\begin{array}{l}\mathrm{C} 21^{(3)}, \mathrm{C} 31^{(7)} \\
\mathrm{D} 1^{(10)} \\
\mathrm{D} 1^{(4)}, \mathrm{D} 1 \mathrm{a}^{(6)} \\
\mathrm{D} 1^{(7)}, \mathrm{D} 1 \mathrm{a}^{(3)} \\
\mathrm{D} 1 \mathrm{a}^{(10)}\end{array}$ & $\begin{array}{l}\mathrm{C} p 8^{(10)} \\
\mathrm{D} p 1^{(10)} \\
\mathrm{D} p 1^{(10)} \\
\mathrm{D} p 1^{(10)} \\
\mathrm{D} p 1^{(10)}\end{array}$ & $\begin{array}{c}\mathrm{Japan}_{2} \\
\text { C31 }\end{array}$ \\
\hline $\begin{array}{l}\text { Agariciidae } \\
\text { Pavona varians }\end{array}$ & $\begin{array}{l}\text { JA-HIMB3 } \\
\text { JA-HIMB3 } \\
\text { JA-HIMB3 } \\
\text { JOH-20 } \\
\text { JOH-4P }\end{array}$ & $\begin{array}{c}\mathrm{C} 1^{(3)}, \mathrm{C} 1 \mathrm{ca}^{(3)}, \mathrm{C} 1 \mathrm{~h}^{(1)}, \mathrm{C} 21.14^{(1)}, \mathrm{C} 45^{(3)} \\
\mathrm{C} 27^{(8)}, \mathrm{C} 27.1^{(2)} \\
\mathrm{C} 1^{(4)}, \mathrm{C} 3^{(2)}, \mathrm{C} 21.14^{(1)}, \mathrm{C} 27^{(2)}, \mathrm{C} 27.1^{(1)}, \mathrm{C} 45^{(3)} \\
\mathrm{C} 27^{(8)}, \mathrm{C} 27.1^{(2)} \\
\mathrm{C} 21^{(1)}, \mathrm{C} 21.14^{(1)}, \mathrm{C} 27^{(7)}, \mathrm{C} 27.1^{(1)}\end{array}$ & $\begin{array}{l}\mathrm{Cp} 1^{(10)} \\
\mathrm{Cp} 1^{(1)}, \mathrm{Cp} p 9^{(9)} \\
\mathrm{C} p 1^{(10)} \\
\mathrm{Cp} 9^{(10)} \\
\mathrm{Cp} 9^{(10)}\end{array}$ & $\begin{array}{l}\text { Pavona: } \\
\text { GBR }_{1,2} \\
\text { C1, C1ca, C27 } \\
\text { Hawaii } \\
\text { C27 }\end{array}$ \\
\hline $\begin{array}{l}\text { Faviidae } \\
\text { Leptastrea sp. }\end{array}$ & $\begin{array}{l}\text { JOH-1AP } \\
\text { JOH-1AP } \\
\text { JOH-1AP } \\
\text { JOH-5P } \\
\text { JOH-5P }\end{array}$ & $\begin{array}{c}\mathrm{C} 1^{(1)}, \mathrm{C} 1.7^{(1)}, \mathrm{C} 1 \mathrm{ca}^{(5)}, \mathrm{C} 1 \mathrm{f}^{(2)}, \mathrm{C} 3^{(1)} \\
\mathrm{C}^{(2)}, \mathrm{C} 1.6^{(1)}, \mathrm{C} 1 \mathrm{ca}^{(2)}, \mathrm{C} 1 \mathrm{f}^{(4)}, \mathrm{C} 3.2^{(1)} \\
\mathrm{C} 1^{(1)}, \mathrm{C} 1 \mathrm{Ca}^{(6)}, \mathrm{C} 1 \mathrm{f}^{(3)} \\
\mathrm{C} 1^{(1)}, \mathrm{C} 1 \mathrm{Ca}^{(5)}, \mathrm{C} 1 \mathrm{f}^{(2)}, \mathrm{C} 3^{(1)}, \mathrm{C} 3.2^{(1)} \\
\mathrm{C} 1^{(1)}, \mathrm{C} 1 \mathrm{Ca}^{(5)}, \mathrm{C} 1 \mathrm{f}^{(1)}, \mathrm{C} 3^{(3)}\end{array}$ & $\begin{array}{l}\mathrm{Cp} 1^{(10)} \\
\mathrm{Cp} 1^{(9)}, \mathrm{Cp} 3^{(1)} \\
\mathrm{C} p 1^{(10)} \\
\mathrm{Cp} 1^{(10)} \\
\mathrm{Cp} 1^{(10)}\end{array}$ & $\begin{array}{l}\text { Leptastrea: } \\
\text { GBR }_{2} \\
\text { C1, C1ca } \\
\text { Hawaii }_{6} \\
\text { C1f }\end{array}$ \\
\hline $\begin{array}{l}\text { Fungiidae } \\
\text { Fungia scutaria }\end{array}$ & $\begin{array}{l}\text { JA-HIMB3 } \\
\text { JOH-4P } \\
\text { JOH-4P } \\
\text { JOH-4P } \\
\text { JOH-5P }\end{array}$ & $\begin{array}{c}\mathrm{C} 1^{(4)}, \mathrm{C} 1 \mathrm{Ca}^{(4)}, \mathrm{C} 1 \mathrm{f}^{(1)}, \mathrm{C} 3^{(1)} \\
\mathrm{C} 1^{(2)}, \mathrm{C} 1.6^{(1)}, \mathrm{C} 1 \mathrm{Ca}^{(5)}, \mathrm{C} 1 \mathrm{f}^{(2)} \\
\mathrm{C} 1.6^{(3)}, \mathrm{C} 1 \mathrm{Ca}^{(6)}, \mathrm{C} 1 \mathrm{f}^{(1)} \\
\mathrm{C} 1^{(2)}, \mathrm{C} 1 \mathrm{ca}^{(4)}, \mathrm{C} 1 \mathrm{f}^{(2)}, \mathrm{C} 3^{(1)}, \mathrm{C} 3.2^{(1)} \\
\mathrm{C} 1^{(7)}, \mathrm{C} 1.7^{(1)}, \mathrm{C} 1.8^{(2)}\end{array}$ & $\begin{array}{l}\mathrm{C} p 1^{(10)} \\
\mathrm{C} p 1^{(10)} \\
\mathrm{C} p 1^{(10)} \\
\mathrm{Cp} 1^{(10)} \\
\mathrm{Cp} 1^{(10)}\end{array}$ & $\begin{array}{l}\text { Fungia: } \\
\mathrm{GBR}_{1,2}, \mathrm{C} 1 \\
\text { Hawaii }_{6}, \mathrm{C} 1 \mathrm{f} \\
\text { Japan }_{2}, \mathrm{C} 1\end{array}$ \\
\hline $\begin{array}{l}\text { Pocilloporidae } \\
\text { Pocillopora } \\
\text { damicornis }\end{array}$ & $\begin{array}{l}\text { JOH-1AP } \\
\text { JOH-23 } \\
\text { JOH-23 } \\
\text { JOH-23 } \\
\text { JOH-25 }\end{array}$ & $\begin{array}{c}\mathrm{C} 1^{(2)}, \mathrm{C} 1.7^{(1)}, \mathrm{C} 42^{(5)}, \mathrm{C} 45^{(2)} \\
\mathrm{C}^{(1)}, \mathrm{C} 1 \mathrm{Ca}^{(2)}, \mathrm{C} 3.12^{(2)}, \mathrm{C} 21^{(1)}, \mathrm{C} 42^{(3)}, \mathrm{C} 45^{(1)} \\
\mathrm{C} 42^{(10)} \\
\mathrm{C} 1.5^{(1)}, \mathrm{C} 3^{(3)}, \mathrm{C} 3.12^{(1)}, \mathrm{C} 42^{(3)}, \mathrm{C} 45^{(3)} \\
\mathrm{C} 3.12^{(2)}, \mathrm{C} 42^{(7)}, \mathrm{C} 45^{(1)}\end{array}$ & $\begin{array}{c}\mathrm{C} p 6^{(10)} \\
\mathrm{Cp} 6^{(10)} \\
\mathrm{Cp} p 1^{(3)}, \mathrm{C} p 6^{(7)} \\
\mathrm{C} p 1^{(4)}, \mathrm{C} p 2^{(1)}, \mathrm{Cp} p 6^{(5)} \\
\mathrm{C} p 1^{(9)}, \mathrm{C} p 6^{(1)}\end{array}$ & $\begin{array}{c}\text { Pocillopora: } \\
\text { AS }_{7} \\
\text { C1, C1c, C42, D, D1a } \\
\text { GBR }_{1,2,4,8} \\
\text { C1, C1ca, C42, C45 }\end{array}$ \\
\hline Pocillopora eydouxi & $\begin{array}{l}\text { JA-HIMB3 } \\
\text { JA-HIMB3 } \\
\text { JA-HIMB3 } \\
\text { JOH-1AP } \\
\text { JOH-22 }\end{array}$ & $\begin{array}{c}\mathrm{A} 1^{(4)}, \mathrm{A} 1.2^{(1)}, \mathrm{C} 1^{(1)}, \mathrm{C} 42^{(4)} \\
\mathrm{A} 1^{(6)}, \mathrm{C} 1^{(1)}, \mathrm{C} 42^{(2)}, \mathrm{C} 45.3^{(1)} \\
\mathrm{A} 1^{(4)}, \mathrm{C} 1.8^{(1)}, \mathrm{C} 42^{(4)}, \mathrm{C} 45.3^{(1)} \\
\mathrm{C} 1^{(2)}, \mathrm{C} 42^{(7)}, \mathrm{C} 45^{(1)} \\
\mathrm{C} 1^{(2)}, \mathrm{C} 42^{(7)}, \mathrm{C} 45^{(1)}\end{array}$ & $\begin{array}{l}\mathrm{A} p 1^{(5)}, \mathrm{A} p 2^{(1)}, \mathrm{C} p 1^{(4)} \\
\mathrm{A} p 1^{(2)}, \mathrm{C} p 1^{(7)}, \mathrm{C} p 3^{(1)} \\
\mathrm{A} p 1^{(2)}, \mathrm{A} p 2^{(4)}, \mathrm{C} p 1^{(4)} \\
\mathrm{C} p 1^{(10)} \\
\mathrm{C} p 1^{(10)}\end{array}$ & $\begin{array}{l}\text { Hawaii }_{6} \\
\text { C45 } \\
\text { Japan }_{2} \\
\text { C1ca, C45 }\end{array}$ \\
\hline $\begin{array}{l}\text { Pocillopora } \\
\text { meandrina }\end{array}$ & $\begin{array}{l}\text { JA-HIMB3 } \\
\text { JA-HIMB3 } \\
\text { JA-HIMB3 } \\
\text { JOH-1AP } \\
\text { JOH-22 }\end{array}$ & $\begin{array}{c}\mathrm{A} 1^{(2)}, \mathrm{C} 1^{(3)}, \mathrm{C} 42^{(1)}, \mathrm{C} 45^{(3)}, \mathrm{D} 1^{(1)} \quad \mathrm{A} p 1 \\
\mathrm{C} 1^{(2)}, \mathrm{C} 42^{(8)} \\
\mathrm{C} 1^{(2)}, \mathrm{C} 42^{(5)}, \mathrm{C} 45^{(3)} \\
\mathrm{D} 1^{(2)}, \mathrm{D} 1 \mathrm{a}^{(8)} \\
\mathrm{C} 1^{(2)}, \mathrm{C} 42^{(3)}, \mathrm{C} 45^{(5)}\end{array}$ & $\begin{array}{c}1^{(1)}, \mathrm{A} p 2^{(6)}, \mathrm{C} p 1^{(2)}, \mathrm{D} p 1 \\
\mathrm{C} p 1^{(10)} \\
\mathrm{C} p 1^{(10)} \\
\mathrm{C} p 1^{(4)}, \mathrm{D} p 1^{(6)} \\
\mathrm{C} p 1^{(10)}\end{array}$ & $1^{(1)}$ \\
\hline $\begin{array}{l}\text { Poritidae } \\
\text { Porites sp. }\end{array}$ & $\begin{array}{l}\text { JOH-5P } \\
\text { JOH-5P } \\
\text { JOH-5P } \\
\text { JOH-5P } \\
\text { JOH-5P }\end{array}$ & $\begin{array}{c}\mathrm{C} 15^{(3)}, \mathrm{C} 15.3^{(1)}, \mathrm{C} 15.4^{(3)}, \mathrm{C} 15.5^{(1)}, \mathrm{C} 15 \mathrm{a}^{(2)} \\
\mathrm{C} 15^{(2)}, \mathrm{C} 15.1^{(2)}, \mathrm{C} 15.4^{(5)}, \mathrm{C} 15.5^{(1)} \\
\mathrm{C} 15^{(2)}, \mathrm{C} 15.1^{(1)}, \mathrm{C} 15.2^{(1)}, \mathrm{C} 15.4^{(6)} \\
\mathrm{C} 15^{(3)}, \mathrm{C} 15.2^{(1)}, \mathrm{C} 15.3^{(1)}, \mathrm{C} 15.4^{(4)}, \mathrm{C} 15 \mathrm{a}^{(1)} \\
\mathrm{C} 15^{(7)}, \mathrm{C} 15.4^{(2)}, \mathrm{C} 15.5^{(1)}\end{array}$ & $\begin{array}{l}\mathrm{Cp} 5^{(10)} \\
\mathrm{Cp} 5^{(10)} \\
\mathrm{Cp} 5^{(10)} \\
\mathrm{Cp} 5^{(10)} \\
\mathrm{Cp} 5^{(10)}\end{array}$ & $\begin{array}{l}\text { Porites: } \\
\quad \mathrm{AS}_{7} \\
\text { C15 } \\
\mathrm{GBR}_{1,2,4} \\
\text { C15 }\end{array}$ \\
\hline Porites lobata & $\begin{array}{l}\text { JOH-1AP } \\
\text { JOH-1AP } \\
\text { JOH-23 } \\
\text { JOH-23 } \\
\text { JOH-23 }\end{array}$ & $\begin{array}{l}\mathrm{C} 15^{(10)} \\
\mathrm{C} 15^{(10)} \\
\mathrm{C} 15^{(10)} \\
\mathrm{C} 15^{(10)} \\
\mathrm{C} 15^{(10)}\end{array}$ & $\begin{array}{l}\mathrm{Cp} 5^{(10)} \\
\mathrm{Cp} 5^{(10)} \\
\mathrm{Cp} 5^{(10)} \\
\mathrm{Cp} 5^{(10)} \\
\mathrm{Cp} 5^{(10)}\end{array}$ & $\begin{array}{l}\text { Hawaii }_{6,9} \\
\text { C15 } \\
\text { Japan }_{2} \\
\text { C15 }\end{array}$ \\
\hline
\end{tabular}


Table 3. GenBank accession numbers for Symbiodinium ITS2 and chloroplast 23S sequences identified in the present study

\begin{tabular}{|c|c|c|c|}
\hline $\begin{array}{l}\text { Sym } \\
\text { Clade }\end{array}$ & $\begin{array}{l}\text { nbiodinium } \\
\text { e Subclade } \\
\text { sequence }\end{array}$ & $\begin{array}{c}\text { Accession } \\
\text { number }\end{array}$ & Source \\
\hline \multicolumn{4}{|l|}{ ITS2 } \\
\hline $\mathrm{A}$ & A1 & AF333505 & LaJeunesse (2001) \\
\hline $\mathrm{A}$ & $\begin{array}{c}\text { A1.2 } \\
\text { (Amami6a) }\end{array}$ & AB207210 & Reimer et al. (2006) \\
\hline $\mathrm{C}$ & $\mathrm{C} 1$ & AF333515 & LaJeunesse (2001) \\
\hline $\mathrm{C}$ & $\begin{array}{c}\text { C1.5 } \\
\text { (DongshanCS) }\end{array}$ & EF634157 & $\begin{array}{l}\text { Z. Dong et al. } \\
\text { unpubl. data }\end{array}$ \\
\hline $\mathrm{C}$ & $\mathrm{C} 1.6$ & FJ461493 & Present study \\
\hline $\mathrm{C}$ & $\mathrm{C} 1.7$ & FJ461494 & Present study \\
\hline $\mathrm{C}$ & $\begin{array}{c}\text { C1.8 } \\
\text { (culture 152) }\end{array}$ & EU074955 & Thornhill et al. (2007) \\
\hline $\mathrm{C}$ & $\begin{array}{c}\mathrm{C} 1 \mathrm{ca} \\
(\mathrm{C} 1 \mathrm{~b} / \mathrm{C} 1 \mathrm{e})\end{array}$ & EU449104 & $\begin{array}{l}\text { T. C. LaJeunesse } \\
\text { unpubl. data }\end{array}$ \\
\hline $\mathrm{C}$ & C1f & AY258490 & LaJeunesse et al. (2004b) \\
\hline $\mathrm{C}$ & $\mathrm{C} 1 \mathrm{~h}$ & AY258473 & LaJeunesse (2005) \\
\hline $\mathrm{C}$ & $\mathrm{C} 3$ & AF499789 & LaJeunesse (2002) \\
\hline $\mathrm{C}$ & $\mathrm{C} 3.2$ & FJ461497 & Present study \\
\hline $\mathrm{C}$ & $\mathrm{C} 3.7$ & FJ461502 & Present study \\
\hline $\mathrm{C}$ & C3.8 & FJ461503 & Present study \\
\hline $\mathrm{C}$ & C3.9 & FJ461504 & Present study \\
\hline $\mathrm{C}$ & C3.10 & FJ461505 & Present study \\
\hline $\mathrm{C}$ & C3.11 & FJ461506 & Present study \\
\hline $\mathrm{C}$ & C3.12 & FJ461507 & Present study \\
\hline $\mathrm{C}$ & $\mathrm{C} 3 \mathrm{~b}$ & AF499791 & LaJeunesse (2002) \\
\hline $\mathrm{C}$ & $\mathrm{C} 15$ & AY239369 & LaJeunesse et al. (2003) \\
\hline $\mathrm{C}$ & C15.1 & FJ461508 & Present study \\
\hline $\mathrm{C}$ & $\mathrm{C} 15.2$ & FJ461509 & Present study \\
\hline $\mathrm{C}$ & C15.3 & FJ461510 & Present study \\
\hline $\mathrm{C}$ & C15.4 & FJ461511 & Present study \\
\hline $\mathrm{C}$ & C15.5 & FJ461512 & Present study \\
\hline $\mathrm{C}$ & $\mathrm{C} 15 \mathrm{a}$ & AY258476 & LaJeunesse (2005) \\
\hline $\mathrm{C}$ & $\mathrm{C} 17$ & AY239370 & LaJeunesse et al. (2003) \\
\hline $\mathrm{C}$ & $\mathrm{C} 17.2$ & FJ461513 & Present study \\
\hline $\mathrm{C}$ & $\mathrm{C} 21$ & EU449102 & LaJeunesse et al. (2003) \\
\hline $\mathrm{C}$ & $\mathrm{C} 21.12$ & FJ461514 & Present study \\
\hline $\mathrm{C}$ & $\mathrm{C} 21.13$ & FJ461515 & Present study \\
\hline $\mathrm{C}$ & $\mathrm{C} 21.14$ & FJ461516 & Present study \\
\hline $\mathrm{C}$ & $\mathrm{C} 27$ & AY239379 & LaJeunesse et al. (2003) \\
\hline $\mathrm{C}$ & $\mathrm{C} 27.1$ & FJ461517 & Present study \\
\hline $\mathrm{C}$ & C31 & AY258496 & LaJeunesse et al. (2004b) \\
\hline $\mathrm{C}$ & $\mathrm{C} 42$ & AY765402 & LaJeunesse (2005) \\
\hline $\mathrm{C}$ & $\mathrm{C} 45$ & EU449103 & LaJeunesse (2005) \\
\hline $\mathrm{C}$ & $\mathrm{C} 45.3$ & FJ461522 & Present study \\
\hline $\mathrm{D}$ & D1 & AF334660 & LaJeunesse (2001) \\
\hline $\mathrm{D}$ & D1a & AF499802z & LaJeunesse (2002) \\
\hline \multicolumn{4}{|l|}{$23 S$} \\
\hline $\mathrm{A}$ & Ap1 & FJ461476 & Present study \\
\hline $\mathrm{A}$ & Ap2 & FJ461477 & Present study \\
\hline $\mathrm{C}$ & $\mathrm{Cp} 1$ & FJ461478 & Present study \\
\hline $\mathrm{C}$ & $\mathrm{Cp} 2$ & FJ461479 & Present study \\
\hline $\mathrm{C}$ & $\mathrm{Cp} 3$ & FJ461480 & Present study \\
\hline $\mathrm{C}$ & $\mathrm{Cp} 4$ & FJ461481 & Present study \\
\hline $\mathrm{C}$ & Cp5 & FJ461482 & Present study \\
\hline $\mathrm{C}$ & Cp6 & FJ461483 & Present study \\
\hline $\mathrm{C}$ & $\mathrm{Cp} 7$ & FJ461484 & Present study \\
\hline $\mathrm{C}$ & $\mathrm{Cp} 8$ & AY035425 & Santos et al. (2002) \\
\hline $\mathrm{C}$ & $\mathrm{Cp} 9$ & FJ461486 & Present study \\
\hline $\mathrm{D}$ & $\mathrm{D} p 1$ & FJ461475 & Present study \\
\hline
\end{tabular}

libraries, respectively. A higher diversity of sequences was recovered using the ITS2 as compared to the chloroplast 23S marker. Two subclade A, 36 subclade C and 2 subclade D sequences were recovered using ITS2 (secondary structures for these ITS2 sequences are given in supplement 1 available at www.int-res.com/articles/ suppl/m386p083_app.pdf) and 2 subclade A, 9 subclade $\mathrm{C}$ and 1 subclade D sequences were recovered with chloroplast 23S (Tables 2 \& 3).

Of the corals sampled, ITS2 sequences representing a single Symbiodinium clade were recovered from $90 \%$ of the colonies (clade $C: \mathrm{n}=47$; clade $\mathrm{D}: \mathrm{n}=7$ ), $8.3 \%$ contained 2 clades (clades C and D in Montipora capitata: $\mathrm{n}=2$; clades $\mathrm{A}$ and $\mathrm{C}$ in Pocillopora eydouxi: $\mathrm{n}=3$ ) and $1.7 \%$ contained 3 clades (clades A, C and D in Pocillopora meandrina: $\mathrm{n}=1$ ). Similarly, chloroplast 23S sequences representing a single Symbiodinium clade were recovered from $88.3 \%$ of the colonies (clade $\mathrm{C}: \mathrm{n}=47$; clade $\mathrm{D}: \mathrm{n}=6$ ), $10 \%$ contained 2 clades (clades $\mathrm{C}$ and $\mathrm{D}$ in $M$. capitata: $\mathrm{n}=2$, and P. meandrina: $\mathrm{n}=1$; clades $\mathrm{A}$ and $\mathrm{C}$ in $P$. eydouxi: $\mathrm{n}=$ 3 ) and $1.7 \%$ contained 3 clades (clades A, C and D in P. meandrina: $\mathrm{n}=1$ ). All coral colonies harbored diverse assemblages of Symbiodinium ITS2 sequences, except for Porites lobata, which contained only C15. In contrast, in the majority of corals where a single Symbiodinium clade was detected, this was represented by a single chloroplast 23S sequence.

Symbiodinium ITS2 and chloroplast 23S sequences resolve into a single network for each clade except for the 2 clade A chloroplast 23S sequences (Fig. 2). The network structure for clade C Symbiodinium is similar between the 2 markers. The ITS2 and chloroplast 23S clade $\mathrm{C}$ networks both show shared sequences present in several coral species that are inferred as the ancestral sequence (C1 for ITS2 and Cp1 for chloroplast 23S) with multiple genus-specific sequences that have evolved from these. Specific Symbiodinium clade C sequences, resolved using both markers, were found associated with the coral genera Porites, Pocillopora, Montipora and Pavona. The coral genus Acropora contained multiple genus-specific Symbiodinium ITS2 sequences in addition to C3. In contrast, a single Acropora-specific Symbiodinium sequence was resolved using the chloroplast 23S rDNA ( $\mathrm{Cp} 4$ ) that was a single base change from the shared dominant sequence type Cp1 harbored by corals with Symbiodinium ITS2 sequences $\mathrm{C} 1, \mathrm{C} 3, \mathrm{C} 1 \mathrm{f}, \mathrm{C} 1 \mathrm{~h}$ and $\mathrm{C} 1 \mathrm{ca}$.

\section{Analyses of Symbiodinium sequence signatures and community signatures}

Cluster analysis of the Symbiodinium sequence signature from each coral colony largely grouped into 

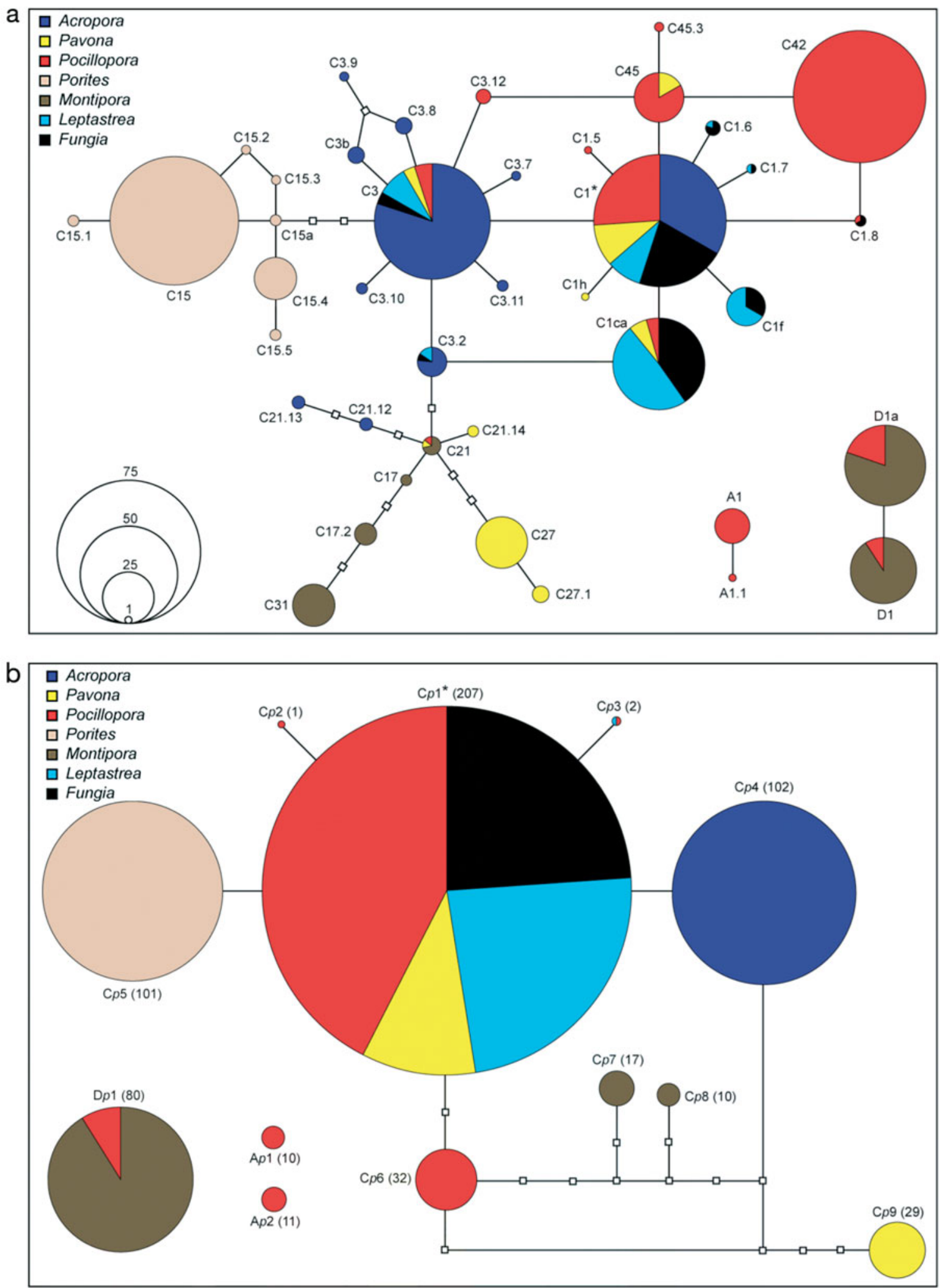

Fig. 2. Statistical parsimony networks for Symbiodinium clades A, C and D for (a) ITS2 and (b) chloroplast 23S sequences retrieved from corals sampled at Johnston Atoll. The size of each pie chart represents the frequency of a given sequence in the corals sampled. Open squares joining pie charts represent a single mutational step and an asterisk denotes the inferred ancestral sequence. Numbers in parentheses indicate the frequency of that sequence in the study 


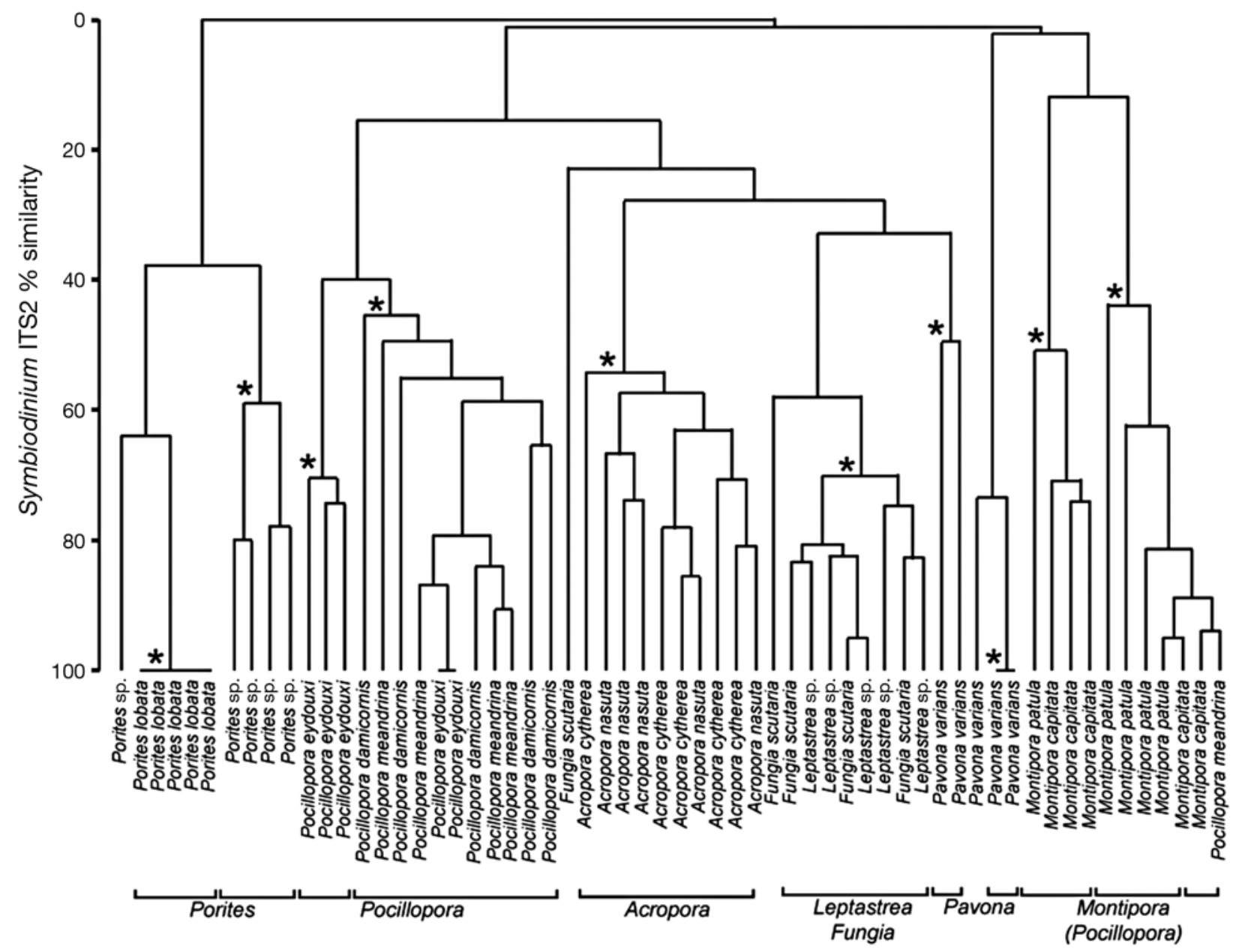

Fig. 3. UPGMA cluster dendrogram of Bray-Curtis similarities calculated from square-root transformed relative abundances of Symbiodinium ITS2 sequences in coral colonies sampled at Johnston Atoll. Asterisks at nodes indicate significant groupings of Symbiodinium ITS2 sequence signatures from the coral colonies $(\mathrm{p}<0.05)$ calculated from the SIMPROF test

species- or genus-specific assemblages for both markers (Figs. 3 \& 4). For example, the Symbiodinum sequence signatures from colonies of Porites grouped into a significant cluster in both the ITS2 and chloroplast 23S dendrograms. Symbiodinium from some coral species (e.g. Leptastrea spp. and Fungia spp.) grouped into a single significant cluster in both the ITS2 and chloroplast 23S dendrograms. The presence of clade D Symbiodinium in coral colonies obscured significant groupings by species or genus in some instances (e.g. Pocillopora meandrina grouping with Montipora). The higher Symbiodinium diversity recovered using the ITS2 compared to the chloroplast 23S marker is also reflected in the higher number of significant clusters identified in the ITS2 compared to the chloroplast 23S dendrogram. For example, the Symbiodinium sequence signatures from colonies of Pocillopora grouped into 3 significant clusters in the ITS2 dendrogram and only 2 in the chloroplast 23S dendrogram.

Differences among the Symbiodinium community sequence signature for coral species sampled at Johnston Atoll resolved using both genetic markers were supported by significant results in the ANOSIM global test (ITS2: $\mathrm{R}=0.770, \mathrm{p}<0.05$; chloroplast 23S: $\mathrm{R}=$ $0.734, \mathrm{p}<0.05)$, and pairwise comparisons showed that the Symbiodinium community sequence signature for most coral species were significantly different from one another (Table 4). Significant differences in the pairwise comparisons of Symbiodinium community sequence signatures were mostly congruent with the hierarchical clustering analysis for significant groupings of Symbiodinium sequence signatures from individual colonies. Overall, these data show that the host taxonomy influences the community of Symbiodinium harbored by corals at Johnston Atoll. 


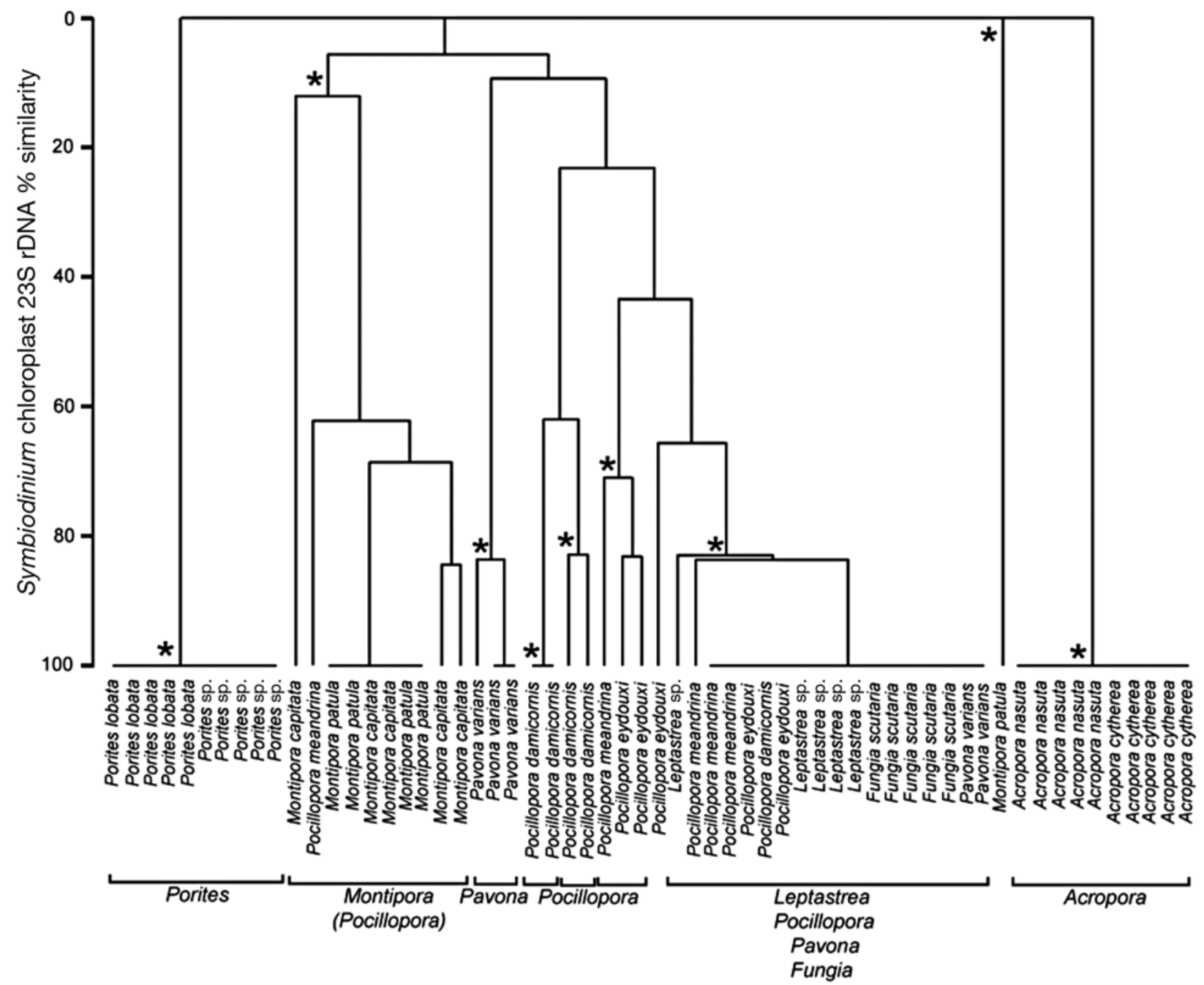

Fig. 4. UPGMA cluster dendrogram of Bray-Curtis similarities calculated from square-root transformed relative abundances of Symbiodinium chloroplast 23S sequences in coral colonies sampled at Johnston Atoll. Asterisks at nodes indicate significant groupings of Symbiodinium chloroplast 23S sequence signatures from the coral colonies $(p<0.05)$ calculated from the SIMPROF test

\section{DISCUSSION}

The diversity of Symbiodinium obtained using the nuclear ITS2 and chloroplast 23S rDNA for corals at Johnston Atoll reveals similar patterns in the hostdinoflagellate assemblages. The large sequence diversity generated with ITS2, much of which can arguably be attributed to intragenomic and/or intraspecific variation, is largely resolved into distinct chloroplast 23S sequences. As such, even though chloroplast $23 \mathrm{~S}$ provides less taxonomic resolution than ITS2, the apparent lack of intragenomic variation makes it easier to interpret and thus a good marker for characterizing Symbiodinium diversity.

\section{Symbiodinium diversity and host specificity at Johnston Atoll}

A high level of Symbiodinium ITS2 diversity was recovered from the coral population at Johnston Atoll, consistent with the findings of Apprill \& Gates (2007). Symbiodinium assemblages are largely species- or genus-specific and those shared among coral species are generally associated with hosts that acquire their Symbiodinium anew each generation from the environment (horizontal acquisition). This is consistent with patterns in the coral-dinoflagellate assemblages in the southern Great Barrier Reef (LaJeunesse et al. 2003, Stat et al. 2008a). Symbiodinium ITS2 types within 


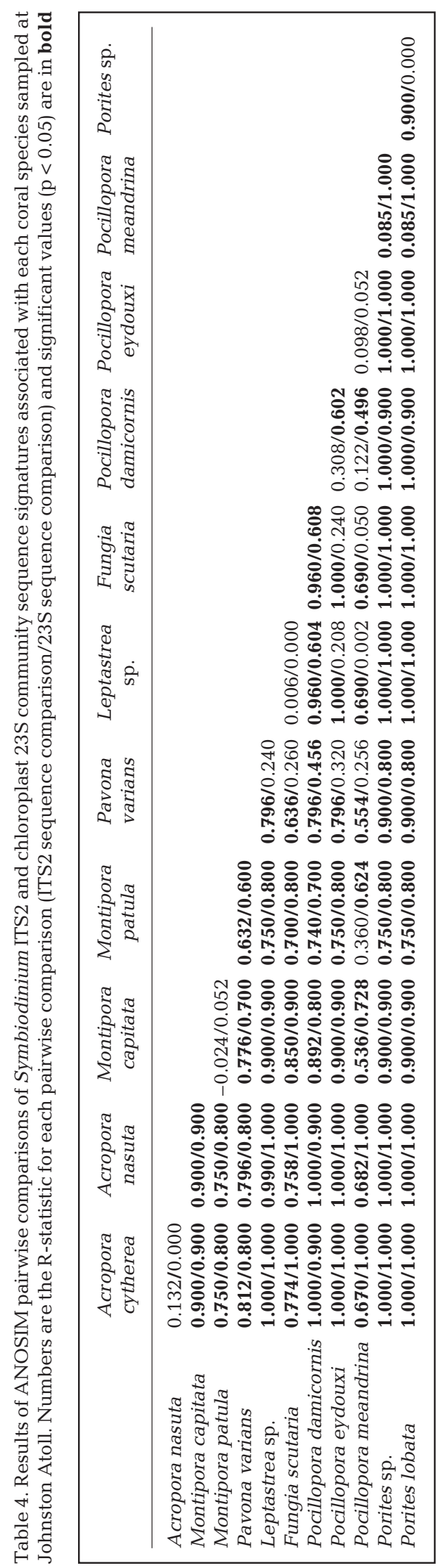

clade $\mathrm{C}$ are thought to have evolved from pandemic generalist types $\mathrm{C} 1$ and $\mathrm{C} 3$ that are found globally and interact with a diverse range of marine invertebrate hosts (LaJeunesse 2005). Here, Symbiodinium C1 was inferred as the ancestral sequence in the statistical parsimony network analysis, with C3 situated a single mutational step from $\mathrm{C} 1$. These 2 sequences were found in multiple host genera including Acropora, Pavona, Pocillopora, Leptastrea and Fungia. Clusters of sequences specific to the coral genera Porites, Pocillopora, Pavona, Montipora and Acropora surround $\mathrm{C} 3, \mathrm{C} 1$ and $\mathrm{C} 1 \mathrm{ca}$. In addition, the most abundant sequences generally occupy a central position within the network, with less common sequences radiating from them, a finding consistent with Thornhill et al. (2007). The chloroplast 23S network reduces the complexity of Symbiodinium diversity by collapsing most of the rare ITS2 sequences into a single sequence type and also combining some abundant ITS2 sequences (e.g. C1, C3 and C1f), while maintaining a network structure largely consistent with that of the ITS2 rDNA. Comparisons of the Symbiodinium community sequence signatures found in each coral species are also similar between the 2 markers, with most forming significant clusters specific to a coral genus or symbiont acquisition strategy.

The coral genera Pocillopora, Porites and Montipora all associate with specific Symbiodinium sequences that are consistent with other studies that used the ITS2 marker (LaJeunesse et al. 2004a,b, LaJeunesse 2005, Sampayo et al. 2007, Stat et al. 2008a). Here, we show that genus-specific lineages for the chloroplast 23S rDNA mimic those of ITS2 rDNA. Also, Pavona and Acropora harbored specific Symbiodinium sequences in addition to the generalist ITS2 C1 that both genera are known to associate with on other Pacific reefs (LaJeunesse et al. 2003, 2004a,b). The extent to which these specific associations occur will be evident as more studies are performed using these markers across different reefs in the Pacific. Although coevolution and reciprocal speciation has not been confirmed between coral and Symbiodinium, the specificity in hostsymbiont associations is likely the result of coadaptation and the evolution of ecologically successful partnerships that have evolved from generalist associations. It is therefore likely that these specific symbiotic partnerships have enabled a functionally advantageous holobiont to become more widespread across the Pacific.

\section{Biogeography of coral-Symbiodinium assemblages}

In addition to most corals harboring multiple Symbiodinium ITS2 sequences, novel sequences, new host-symbiont assemblages and/or new biogeogra- 
phic patterns in Symbiodinium were revealed. Comparison of the host-symbiont assemblages described here using chloroplast $23 \mathrm{~S}$ is limited due to the low number of studies that have used this marker in the past, and hence the only shared sequence is that between Symbiodinium from Pocillopora damicornis from Johnston Atoll and a culture originating from an unidentified anemone from Japan (Santos et al. 2002). A comparison of the patterns in host-symbiont assemblages at Johnston Atoll with other reefs in the Pacific described using ITS2 reveals both similarities and differences. For example, Acropora cytherea at Johnston Atoll associates with Symbiodinium ITS2 C1, C3 and C3b, while in the Northwestern Hawaiian Islands, the closest reef system to Johnston Atoll, A. cytherea associates with A1, C1c and C27 (Stat \& Gates 2008) and in the Great Barrier Reef with just C3 (LaJeunesse et al. 2003, 2004a). Similarly, A. nasuta harbors C3 and C3b at Johnston Atoll, but in the Great Barrier Reef associates with C3, C3k and C3i (LaJeunesse et al. 2003, 2004b). Symbiodinium ITS2 C3b has also previously been reported as Caribbean-specific (LaJeunesse 2005). Other novel symbioses recovered at Johnston Atoll include C21, C3, A1 and D1a with Pocillopora, C1h, C3 and C45 with Pavona, C3 with Leptastrea and C3 with Fungia. The novel assemblages identified for Johnston Atoll and differences in the Symbiodinium assemblages with locations in the Pacific likely reflect specificity driven by geographic location and isolation, local host-symbiont adaptation and fine-scale niche partitioning of symbionts.

\section{Intragenomic nature of rDNA and analyses using community signatures}

Intragenomic variation in rDNA is common in dinoflagellates and other single-celled protists (Litaker et al. 2003, 2007, Aktas et al. 2007, Thornhill et al. 2007). Furthermore, in Symbiodinium, ITS2 sequence divergence within a genome has been found to be greater than that between sequences labeled as ecologically distinct types (van Oppen et al. 2001, Thornhill et al. 2007). This makes distinguishing between intragenomic, interspecific and intraspecific variation in the ITS2 sequences recovered from a single coral extremely challenging. Also, a rare sequence variant in one Symbiodinium cell may be the dominant copy in a different cell, making it difficult to determine the ecological importance of these ITS2 variants. For example, Symbiodinium ITS2 C1 and C3 (the inferred ancestral sequences in clade C) are dominant types in a wide range of corals, but these sequences are likely present in many different cells where the dominant rDNA copy has evolved to a different sequence (e.g. C42). These interpretational problems are inherent when working with a multicopy marker.

Identifying dominant Symbiodinium ITS2 sequences within a coral using DGGE analysis has been used as a method to screen for the putative ecologically significant type (e.g. LaJeunesse 2002, LaJeunesse et al. 2003, 2004a,b, Thornhill et al. 2006a,b, Pochon et al. 2007, Sampayo et al. 2007, Stat et al. 2008a). This methodology has been widely used and has significantly increased our understanding of Symbiodinium diversity and biogeography. However, detection limits of the technique and interpretation of DGGE banding profiles can underestimate diversity (Thornhill et al. 2006b, Stat et al. 2008a) and misidentification of ITS2 types due to comigration of different sequences can occur (Apprill \& Gates 2007, Pochon et al. 2007, Sampayo et al. 2008).

In the present study we used an extremely conservative approach to selecting cloned sequence data to describe the Symbiodinium diversity in individual corals and used statistical treatments to detect patterns in the assemblages of Symbiodinium associated with different coral species and genera. This analysis resolved high ITS2 sequence diversity that formed significantly different groupings by host species or genus. This approach circumvents the need to assign the ecological importance of a given sequence by naming it a type and more accurately reflects the complexity of the rDNA and the heterogeneity of coral-dinoflagellate symbiosis. However, exploring Symbiodinium diversity in coral hosts would be made much easier using a marker with no intragenomic variation.

With this in mind, we explored the diversity of Symbiodinium sequences recovered using chloroplast 23S rDNA in parallel with ITS2. Lower sequence diversity was recovered using chloroplast 23S rDNA as compared to ITS2 (usually a single Symbiodinium sequence per coral host), but the patterns in host-symbiont assemblages were still detectable. This marker is considered diverse enough to delineate species (Barbrook et al. 2006), and although multiple copies of the chloroplast 23S plastid minicircle are present in dinoflagellates and the copy number changes with the growth phase of the cell, the transcribed region appears homogenous within cells (Zhang et al. 2002, Koumandou \& Howe 2007). The data presented here certainly support the broader application of chloroplast 23S rDNA as an easily interpretable and informative marker for assessing Symbiodinium diversity.

In conclusion, corals from Johnston Atoll in the central Pacific host diverse Symbiodinium communities that are specific to host species and genera. Some coral species conform to the Pacific-wide pattern of general and specific symbioses, while others engage in novel unions with Symbiodinium. The chloroplast 23S mar- 
ker resolves most of the patterns in host-symbiont assemblages detected with ITS2 and exhibits lower intragenomic variation, making the Symbiodinium diversity data collected using this marker much easier to interpret. These findings set the stage to explore the functional implications of the distinct patterns in Symbiodinium diversity found in corals from Johnston Atoll and provide ample rationale to more broadly apply chloroplast 23S in studies of Symbiodinium diversity.

Acknowledgements. The authors thank B. Wheeler for his assistance in the field, M. J. Huggett for her help with statistics, the captain and crew of the NOAA RV 'Hi'ialakai', and 3 anonymous reviewers. Coral samples were collected under the permit DNLR.NWH106R006 issued to R.D.G, and the research supported through funding from the National Marine Sanctuary Program and Hawaii Institute of Marine Biology Reserve Partnership (memorandum of agreement 2005-008/66882) and a National Science Foundation Award OCE-0752604 to R.D.G. We also thank the Swiss National Science Foundation (PBGEA-115118 to X.P.) and the School of Ocean and Earth Science and Technology at the University of Hawaii for financial support. This is Hawaii Institute of Marine Biology contribution no. 1346.

\section{LITERATURE CITED}

Adachi M, Sako Y, Ishida Y (1996) Analysis of Alexandrium (Dinophyceae) species using sequences of the 5.8S ribosomal DNA and internal transcribed spacer regions. J Phycol 32:424-432

Aktas M, Bendele KG, Altay K, Dumanli N, Tsuji M, Holman PJ (2007) Sequence polymorphism in the ribosomal DNA internal transcribed spacers differs among Theileria species. Vet Parasitol 147:221-230

Apprill AM, Gates RD (2007) Recognizing diversity in coral symbiotic dinoflagellate communities. Mol Ecol 16: 1127-1134

Baker AC, Rowan R, Knowlton N (1997) Symbiosis ecology of two Caribbean Acroporid corals. Proc 8th Int Coral Reef Symp 2:1295-1300

- Baker AC, Starger CJ, McClanahan TR, Glynn PW (2004) Coral reefs: corals' adaptive response to climate change. Nature 430:741

Barbrook AC, Santucci N, Plenderleith LJ, Hiller RG, Howe CJ (2006) Comparative analysis of dinoflagellate chloroplast genomes reveals rRNA and tRNA genes. BMC Genomics 7:297

Bray JR, Curtis JT (1957) An ordination of the upland forest communities of southern Wisconsin. Ecol Monogr 27: 325-349

> Cantacessi C, Riddell S, Morris GM, Doran T, Woods WG, Otranto D, Gasser RB (2008) Genetic characterization of three unique operational taxonomic units of Eimeria from chickens in Australia based on nuclear spacer ribosomal DNA. Vet Parasitol 152:226-234

> Carlos AA (1999) Phylogenetic position of Symbiodinium (Dinophyceae) isolates from tridacnids (Bivalvia), cardiids (Bivalvia), a sponge (Porifera), a soft coral (Anthozoa), and a free-living strain. J Phycol 35:1054-1062

> Chen D, Wang G, Tao W, Nie P (2007) Utility of ITS15.8S-ITS2 sequences for species discrimination and phylogenetic inference of two closely related bucephalid digeneans (Digenea: Bucephalidae): Dollfustrema vaneyi and Dollfustrema hefeiensis. Parasitol Res 101:791-800

Clarke KR, Warwick RM (2001) Change in marine communities: an approach to statistical analysis and interpretation, 2nd edn. PRIMER-E, Plymouth

Clement M, Posada D, Crandall KA (2000) TCS: a computer program to estimate gene genealogies. Mol Ecol 9: $1657-1659$

Coffroth MA, Santos SR (2005) Genetic diversity of symbiotic dinoflagellates in the genus Symbiodinium. Protist 156: 19-34

Coleman AW (2003) ITS2 is a double-edged tool for eukaryotic evolutionary comparisons. Trends Genet 19:370-375

Correa AMS, Baker AC (2009) Understanding diversity in coral-algal symbiosis: a cluster-based approach to interpreting fine-scale genetic variation in the genus Symbiodinium. Coral Reefs 28:81-93

Correa AMS, Brandt ME, Smith TB, Thornhill DJ, Baker AC (2009) Symbiodinium associations with diseased and healthy scleractinian corals. Coral Reefs 28:437-448

Denboh T, Ichimura T, Hendrayanti D, Coleman AW (2003) Closterium moniliferum-ehrenbergii (Charophyceae, Chlorophyta) species complex viewed from the 1506 group 1 intron and ITS2 of nuclear rDNA. J Phycol 39: 960-977

Godhe A, McQuoid MR, Karunsagar I, Karunsagar I, Rehnstam-Holm AS (2006) Comparison of three common molecular tools for distinguishing among geographically separated clones of the diatom Skeletonema marinoi sarno et zingone (Bacillariophyceae). J Phycol 42:280-291

Gray MW (1999) Evolution of organellar genomes. Curr Opin Genet Dev 9:678-687

Green BR (2004) The chloroplast genome of dinoflagellates: A reduced instruction set? Protist 155:23-31

Herzog M, Maroteaux L (1986) Dinoflagellate 17S rRNA sequence inferred from the gene sequence: evolutionary implications. Proc Natl Acad Sci USA 83:8644-8648

Hunter RL, LaJeunesse TC, Santos SR (2007) Structure and evolution of the rDNA internal transcribed spacer (ITS) region 2 in the symbiotic dinoflagellates (Symbiodinium, Dinophyta). J Phycol 43:120-128

Iglesias-Prieto R, Beltran VH, LaJeunesse TC, Reyes-Bonilla $H$, Thorne PE (2004) Different algal symbionts explain the vertical distribution of dominant reef corals in the Eastern Pacific. Proc R Soc Lond B Biol Sci 271:1757-1763

Kawahata M, Fujii T, Lefuji H (2007) Intraspecies diversity of the industrial yeast strains Saccharomyces cerevisia and Saccharomyces pastorianus based on analysis of the sequences of the internal transcribed spacer (ITS) regions and the D1/D2 region of 26S rDNA. Biosci Biotechnol Biochem 71:1616-1620

> Koumandou VL, Howe CJ (2007) The copy number of chloroplast gene minicircles changes dramatically with growth phase in the dinoflagellate Amphidinium operculatum. Protist 158:89-103

> LaJeunesse TC (2001) Investigating the biodiversity, ecology, and phylogeny of endosymbiotic dinoflagellates in the genus Symbiodinium using the ITS region: in search of a 'species' level marker. J Phycol 37:866-880

LaJeunesse TC (2002) Diversity and community structure of symbiotic dinoflagellates from Caribbean coral reefs. Mar Biol 141:387-400

> LaJeunesse TC (2005) 'Species' radiations of symbiotic dinoflagellates in the Atlantic and Indo-Pacific since the Miocene-Pliocene transition. Mol Biol Evol 22:570-581

$>$ LaJeunesse TC, Trench RK (2000) Biogeography of two species of Symbiodinium (Freudenthal) inhabiting the inter- 
tidal sea anemone Anthopleura elegantissima (Brandt). Biol Bull 199:126-134

LaJeunesse TC, Loh WKW, van Woesik R, Hoegh-Guldberg O, Schmidt GW, Fitt WK (2003) Low symbiont diversity in southern Great Barrier Reef corals, relative to those of the Caribbean. Limnol Oceanogr 48:2046-2054

LaJeunesse TC, Bhagooli R, Hidaka M, deVantier L and others (2004a) Closely related Symbiodinium spp. differ in relative dominance in coral reef host communities across environmental, latitudinal and biogeographic gradients. Mar Ecol Prog Ser 284:147-161

LaJeunesse TC, Thornhill DJ, Cox EF, Stanton FG, Fitt WK, Schmidt GW (2004b) High diversity and host specificity observed among symbiotic dinoflagellates in reef communities from Hawaii. Coral Reefs 23:596-603

> LaJeunesse TC, Loh W, Trench RK (2009) Do introduced endosymbiotic dinoflagellates take to new hosts? Biol Invasions 11:995-1003

Le Blancq SM, Khramtsov NV, Zamani F, Upton SJ, Wu TW (1997) Ribosomal RNA gene organization in Cryptosporidium parvum. Mol Biochem Parasitol 90:463-478

Litaker RW, Vandersea MW, Kibler SR, Reece KS and others (2003) Identification of Pfiesteria piscicida (Dinophyceae) and Pfiesteria-like organisms using internal transcribed spacer-specific PCR assays. J Phycol 39: 754-761

Litaker RW, Vandersea MW, Kibler SR, Reece KS and others (2007) Recognizing dinoflagellate species using ITS rDNA sequences. J Phycol 43:344-355

Little AF, van Oppen MJH, Willis BL (2004) Flexibility in algal endosymbioses shapes growth in reef corals. Science 304:1492-1494

Lobban CS, Schefter M, Simpson AGB, Pochon X, Pawlowski J, Foissner W (2002) Maristentor dinoferus n. gen., n. sp., a giant heterotrich ciliate (Spirotrichea: Heterotrichida) with zooxanthellae, from coral reefs on Guam, Mariana Islands. Mar Biol 140:411-423

Long EO, Dawid IB (1980) Repeated genes in eukaryotes. Annu Rev Biochem 49:727-764

Lott TJ, Burns BM, Zancope-Oliveira R, Elie CM, Reiss E (1998) Sequence analysis of the internal transcribed spacer 2 (ITS2) from yeast species within the genus Candida. Curr Microbiol 36:63-69

Muscatine L, McCloskey LR, Marian RE (1981) Estimating the daily contribution of carbon from zooxanthellae to coral animal respiration. Limnol Oceanogr 26:601-611

>rsini L, Procaccini G, Sarno D, Montresor M (2004) Multiple rDNA ITS-types within the diatom Pseudo-nitzschia delicatissima (Bacillariophyceae) and their relative abundances across a spring bloom in the Gulf of Naples. Mar Ecol Prog Ser 271:87-98

Pawlowski J, Holzmann M, Fahrni JF, Pochon X, Lee JJ (2001) Molecular identification of algal endosymbionts in large miliolid foraminifera: 2. Dinoflagellates. J Eukaryot Microbiol 48:368-373

Pochon X, Pawlowski J, Zaninetti L, Rowan R (2001) High genetic diversity and relative specificity among Symbiodinium-like endosymbiotic dinoflagellates in soritid foraminiferans. Mar Biol 139:1069-1078

Pochon X, LaJeunesse TC, Pawlowski J (2004) Biogeographic partitioning and host specialization among foraminiferan dinoflagellate symbionts (Symbiodinium; Dinophyta). Mar Biol 146:17-27

> Pochon X, Montoya-Burgos JI, Stadelmann B, Pawlowski J (2006) Molecular phylogeny, evolutionary rates, and divergence timing of the symbiotic dinoflagellate genus Symbiodinium. Mol Phylogenet Evol 38:20-30
Pochon X, Garcia-Cuetos L, Baker AC, Castella E, Pawlowski J (2007) One-year survey of a single Micronesian reef reveals extraordinarily rich diversity of Symbiodinium types in soritid foraminifera. Coral Reefs 26:867-882

Reimer JD, Takishita K, Ono S, Maruyama T, Tsukahara J (2006) Latitudinal and intracolony ITS-rDNA sequence variation in the symbiotic dinoflagellate genus Symbiodinium (Dinophyceae) in Zoanthus sansibaricus (Anthozoa: Hexacorallia). Phycol Res 54:122-132

Rodriguez-Lanetty M, Loh W, Carter D, Hoegh-Guldberg O (2001) Latitudinal variability in symbiont specificity within the widespread scleractinian coral Plesiastrea versipora. Mar Biol 138:1175-1181

> Rowan R (2004) Thermal adaptations in reef coral symbionts. Nature 430:742

Rowan R, Knowlton N (1995) Intraspecific diversity and ecological zonation in coral-algal symbiosis. Proc Natl Acad Sci USA 92:2850-2853

Rowan R, Powers DA (1991) A molecular genetic classification of zooxanthellae and the evolution of animal-algal symbioses. Science 251:1348-1351

Rowan R, Knowlton N, Baker A, Jara J (1997) Landscape ecology of algal symbionts creates variation in episodes of coral bleaching. Nature 388:265-269

Rynearson TA, Armbrust VE (2004) Genetic differentiation among populations of the planktonic marine diatom Ditylum brightwellii (Bacillariophyceae). J Phycol 40:34-43

Sampayo EM, Franceschinis L, Hoegh-Guldberg O, Dove S (2007) Niche partitioning of closely related symbiotic dinoflagellates. Mol Ecol 16:3721-3733

Sampayo EM, Ridgeway T, Bongaerts P, Hoegh-Guldberg O (2008) Bleaching susceptibility and mortality of corals are determined by fine-scale differences in symbiont type. Proc Natl Acad Sci USA 105:10444-10449

Sampayo EM, Dove S, LaJeunesse TC (2009) Cohesive molecular genetic data delineate species diversity in the dinoflagellates genus Symbiodinium. Mol Ecol 18:500-519

Santos SR, Taylor DJ, Kinzie RA III, Hidaka M, Sakai K, Coffroth MA (2002) Molecular phylogeny of symbiotic dinoflagellates inferred from partial chloroplast large subunit (23S)-rDNA sequences. Mol Phylogenet Evol 23: 97-111

> Schultz J, Müller T, Achtziger M, Seibel PN, Dandekar T, Wolf $M$ (2006) The internal transcribed spacer 2 database-a web server for (not only) low level phylogenetic analyses. Nucleic Acids Res 34:W704-W707

Seibel PN, Müller T, Dandekar T, Schultz J, Wolf M (2006) 4SALE: a tool for synchronous RNA sequence and secondary structure alignment and editing. BMC Bioinformatics 7:498

Seibel PN, Müller T, Dandekar T, Wolf M (2008) Synchronous visual analysis and editing of RNA sequence and secondary structure alignments using 4SALE. BMC Res Notes 1:91

Selig C, Wolf M, Müller T, Dandekar T, Schultz J (2008) The ITS2 Database II: homology modelling RNA structure for molecular systematics. Nucleic Acids Res 36:D377-D380

Shankle AM, Mayali X, Franks PJS (2004) Temporal patterns in population genetic diversity of Prorocentrum micans (Dinophyceae). J Phycol 40:239-247

Smith LW, Wirshing HH, Baker AC, Birkeland C (2008) Environmental versus genetic influences on growth rates of the corals Pocillopora eydouxi and Porites lobata (Anthozoa: Scleractinia). Pac Sci 62:57-69

Stat M, Gates RD (2008) Vectored introductions of marine endosymbiotic dinoflagellates into Hawaii. Biol Invasions 10:579-583 
Stat M, Carter D, Hoegh-Guldberg O (2006) The evolutionary history of Symbiodinium and scleractinian hosts: symbiosis, diversity, and the effect of climate change. Perspect Plant Ecol Evol Syst 8:23-43

Stat M, Loh WKW, Hoegh-Guldberg O, Carter DA (2008a) Symbiont acquisition strategy drives host-symbiont associations in the southern Great Barrier Reef. Coral Reefs 27:763-772

Stat M, Morris E, Gates RD (2008b) Functional diversity in coral-dinoflagellate symbiosis. Proc Natl Acad Sci USA 105:9256-9261

Tchernov D, Gorbunov MY, de Vargas C, Narayan Yadav S, Milligan AJ, Häggblom M, Falkowski PG (2004) Membrane lipids of symbiotic algae are diagnostic of sensitivity to thermal bleaching in corals. Proc Natl Acad Sci USA 101:13531-13535

Thornhill DJ, Fitt WK, Schmidt GW (2006a) Highly stable symbiosis among western Atlantic brooding corals. Coral Reefs 25:515-519

Thornhill DJ, LaJeunesse TC, Kemp DW, Fitt WK, Schmidt GW (2006b) Multi-year, seasonal genotypic surveys of coral-algal symbioses reveal prevalent stability or postbleaching reversion. Mar Biol 148:711-722

Thornhill DJ, LaJeunesse TC, Santos SR (2007) Measuring rDNA diversity in eukaryotic microbial systems: how intragenomic variation, pseudogenes, and PCR arti-

Editorial responsibility: Peter Edmunds,

Northridge, California, USA facts confound biodiversity estimates. Mol Ecol 16:5326-5340

Ulstrup KE, van Oppen MJH (2003) Geographic and habitat partitioning of genetically distinct zooxanthellae (Symbiodinium) in Acropora corals on the Great Barrier Reef. Mol Ecol 12:3477-3484

van Oppen MJH, Palstra FP, Piquet MT, Miller DJ (2001) Patterns of coral-dinoflagellate associations in Acropora: significance of local availability and physiology of Symbiodinium strains and host-symbiont selectivity. Proc R Soc Lond B Biol Sci 268:1759-1767

van Oppen MJH, Mahiny AJ, Done TJ (2005) Geographic distribution of zooxanthella types in three coral species on the Great Barrier Reef sampled after the 2002 bleaching event. Coral Reefs 24:482-487

$>$ Zhang Z, Green BR, Cavalier-Smith T (1999) Single gene circles in dinoflagellate chloroplast genomes. Nature 400: $155-159$

Zhang Z, Green BR, Cavalier-Smith T (2000) Phylogeny of ultra-rapidly evolving dinoflagellate chloroplast genes: a possible common origin for sporozoan and dinoflagellate plastids. J Mol Evol 51:26-40

> Zhang Z, Cavalier-Smith T, Green BR (2002) Evolution of dinoflagellate unigenic minicircles and the partially concerted divergence of their putative replicon origins. Mol Biol Evol 19:489-500

Submitted: November 17, 2008; Accepted: April 23, 2009 Proofs received from author(s): June 28, 2009 